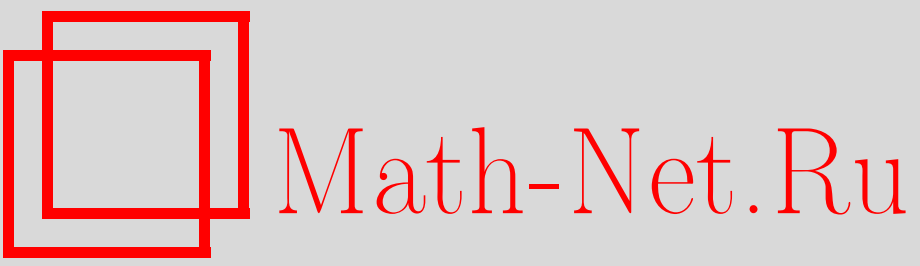

Б. Пеллони, О корректных граничных задачах для интегрируемых эволюционных уравнений на конечном интервале, ТМФ, 2002, том 133, номер 2, 327-336

DOI: https://doi.org/10.4213/tmf401

Использование Общероссийского математического портала Math-Net.Ru подразумевает, что вы прочитали и согласны с пользовательским соглашением

http://www . mathnet.ru/rus/agreement

Параметры загрузки:

IP: 54.197 .130 .99

26 апреля 2023 г., 07:19:34 
ТЕОРЕТИЧЕСКАЯ

И МАТЕМАТИЧЕСКАЯ

ФИЗИКА

Том 133, № 2

ноябрь, 2002

(C) 2002 г.

Б. Пеллони*

\section{О КОРРЕКТНЫХ ГРАНИЧНЫХ ЗАДАЧАХ ДЛЯ ИНТЕГРИРУЕМЫХ ЭВОЛЮЦИОННЫХ УРАВНЕНИЙ НА КОНЕЧНОМ ИНТЕРВАЛЕ}

Рассматриваются граничные задачи для произвольного заданного на интервале $[0, L]$ линейного эволюционного уравнения в одномерном пространстве с пространственными производными порядка $n$. Класс подобных задач характеризуется тем, что они допускают единственное решение и в этом смысле являются корректно поставленными. Такие корректно поставленные граничные задачи получаются путем предписывания $N$ условий при $x=0$ и $n-N$ условий при $x=L$, где $N$ зависит от $n$ и от знака коэффициента при старшей степени $\alpha_{n}$ в дисперсионном соотношении для данного уравнения. Для задач такого класса дается интегральное представление решения в виде спектрального разложения; более того, показывается, что подобные задачи являются единственными задачами, допускающими такое представление. Полученные результаты можно использовать для установления корректной определенности, по крайней мере локально по времени, некоторых физически актуальных нелинейных эволюционных уравнений в одномерном пространстве.

Ключевые слова: граничные задачи, задача Римана-Гильберта, спектральный анализ.

\section{1. ВВЕДЕНИЕ}

В недавних работах [1], [2] метод преобразования Фокаса [3] был применен к решению класса двухточечных граничных задач для общего линейного эволюционного уравнения с дисперсией вида

$$
\left(\partial_{t}+i \sum_{j=0}^{n} \alpha_{j}\left(-i \partial_{x}\right)^{j}\right) q(x, t)=0, \quad 0<x<L, \quad 0<t<T
$$

где $q(x, t)$ - некоторая вещественная функция, $\alpha_{j} \in \mathbb{R}$ - константы, $\alpha_{n} \neq 0, L$ и $T$ - положительные константы. В настояшей работе мы предполагаем, что $q(x, t)$ также удовлетворяет начальному условию

$$
q(x, 0)=q_{0}(x), \quad 0<x<L,
$$

\footnotetext{
${ }^{*}$ Department of Mathematics, University of Reading, Reading, UK. E-mail: b.pelloni@rdg.ac.uk
} 
где $q_{0}(x)$ - достаточно гладкая функция. Дисперсионное соотношение для уравнения (1.1) имеет вид

$$
\omega(k)=\sum_{j=1}^{n} \alpha_{j} k^{j} .
$$

Вообще говоря, в предположении существования решения метод Фокаса дает алгоритм построения интегрального представления этого решения. Это представление несет явную экспоненциальную зависимость от переменных $x$ и $t$ и включает некоторую $c n e \kappa m-$ ральную функиию, определенную в терминах всех начальных и граничных значений решения. В случае конечного интервала это представление может также иметь альтернативную форму, которая содержит бесконечную сумму, соответствующую точкам дискретного спектра.

Коль скоро такое представление получено, проблема состоит в том, чтобы выразить спектральную функцию в терминах только начального условия и заданных граничных условий, а не всех граничных значений. Если задача является корректной, этого можно достигнуть путем использования некоторого глобального уравнения, связывающего все граничные значения решения. Используя этот факт, можно строго доказать сушествование решения поставленной задачи.

Результаты, полученные в двух упомянутых работах, можно суммировать следующим образом.

ТЕОРема 1.1. Рассмотрим граничную задачу для уравнения (1.1) при заданных начальном условии (1.2) и граничных условиях

$$
u_{j_{1}}(t)=\left(-i \partial_{x}\right)^{j_{1}} q(0, t), \quad v_{j_{2}}(t)=\left(-i \partial_{x}\right)^{j_{2}} q(L, t),
$$

где $\left(j_{1}, j_{2}\right) \in J_{1} \times J_{2}$ и $J_{i}, \quad i=1,2,-$ подмножсества множества индексов $\{0, \ldots$ $\ldots, n-1\}$.

Предположим, что әти начальные и граничные условия принадлежат классу $\mathbf{C}^{\infty}$ и что они совместны, т.е. $\left(-i \partial_{x}\right)^{j_{1}} q_{0}(0)=u_{j_{1}}(0), j_{1} \in J_{1}, u\left(-i \partial_{x}\right)^{j_{2}} q_{0}(L)=$ $v_{j_{2}}(0), j_{2} \in J_{2}$.

Если $\left|J_{1}\right|=N$ и $\left|J_{2}\right|=n-N$, где $|\cdot|$ обозначает мощность множества индексов, а $N$ имеет вид

$$
N=\left\{\begin{array}{lll}
n / 2, & n \text {-четное }, \\
(n+1) / 2, & n-\text {-ечетное, } & \alpha_{n}>0, \\
(n-1) / 2, & n-\text { - еегтное, } & \alpha_{n}<0,
\end{array}\right.
$$

то указанная граничная задача имеет единственное решение $q(x, t)$ такое, что отображсение $t \rightarrow q(\cdot, t)$ является $\mathbf{C}^{\infty}$-отображением из $[0, T]$ в $\mathbf{C}^{\infty}[0, L]$.

Можно показать, что задачи, описываемые в утверждении теоремы 1.1 , являются единственными задачами с граничными условиями, для которых метод Фокаса дает такой результат. Это связано с тем, что если $\left|J_{1}\right| \neq N$ или $\left|J_{2}\right| \neq n-N$, то, даже предполагая сушествование решения, при попытке выразить его представление в терминах только предписанных данных мы получаем спектральную функцию без требуемых свойств ограниченности и аналитичности. 
Это является весомым указанием (хотя и не доказательством) на то, что граничные задачи, отличные от рассмотренных в теореме 1.1, не являются корректно поставленными. Аналитическому и численному исследованию справедливости этого утверждения будет посвяшена наша последуюшая работа. Для целей, которые мы ставим в настояшей работе, суммируем наше утверждение в виде следуюшего предложения.

ПРЕДЛОЖЕНИЕ 1.1. Рассмотрим задачу с граничными условиями (1.1), (1.2), (1.4) и предположим, что она имеет единственное гладкое решение. Если $\left|J_{1}\right| \neq$ $N$ или $\left|J_{2}\right| \neq n-N$, невозможкно выразить это решение в виде спектрально разложенного интеграла в терминах только начального условия и значений, положенных на границах $x=0$ u $x=L$.

\section{2. ПРЕДСТАВЛЕНИЕ РЕШЕНИЯ}

Рассмотрим уравнение (1.1) и предположим, что оно допускает единственное гладкое решение $q(x, t)$. Чтобы получить интегральное представление этого решения в виде спектрального разложения, нам преж де всего необходимо записать следующее определение.

ОПРЕДЕЛЕНИЕ 2.1. Пусть $D, D_{+}$и $D_{-}-$области в $k$-комплексной плоскости

$$
D=\{k \in \mathbb{C}: \operatorname{Im} \omega(k) \geqslant 0\}, \quad D_{+}=D \cap \mathbb{C}^{+}, \quad D_{-}=D \cap \mathbb{C}^{-},
$$

где $\mathbb{C}^{+}$и $\mathbb{C}^{-}$обозначают соответственно верхнюю и нижнюю полуплоскости. Пусть $\partial D_{+}, \partial D_{-}$являются ориентированными границами областей $D_{+}, D_{-}$, причем $D$ расположена слева от направления возрастания $\partial D$.

ПрЕДЛОЖЕНИЕ 2.1. Предположим, что $q(x, t)$ - достаточно гладкое решение уравнения (1.1). Тогда $q(x, t)$ имеет вид

$$
\begin{aligned}
q(x, t)=\frac{1}{2 \pi} & \left\{\int_{-\infty}^{\infty} \mathrm{e}^{i k x-i \omega(k) t} \hat{q}_{0}(k) d k+\right. \\
& \left.+\int_{\partial D_{+}} \mathrm{e}^{i k x-i \omega(k) t} \widehat{Q}(0, k) d k+\int_{\partial D_{-}} \mathrm{e}^{i k(x-L)-i \omega(k) t} \widehat{Q}(L, k) d k\right\},
\end{aligned}
$$

$2 \partial e$

$$
\hat{q}_{0}(k)=\int_{0}^{L} \mathrm{e}^{-i k x} q(x, 0) d x, \quad k \in \mathbb{C},
$$

а спектральные функции $\widehat{Q}(0, k), \widehat{Q}(L, k)$ определяются выражсениями

$$
\begin{aligned}
\widehat{Q}(\alpha, k) & =\sum_{j=1}^{n} \alpha_{j}\left(\widehat{Q}_{j-1}(\alpha, k)+k \widehat{Q}_{j-2}(\alpha, k)+\cdots+k^{j-1} \widehat{Q}_{0}(\alpha, k)\right), \\
\widehat{Q}_{j}(\alpha, k) & =\int_{0}^{T} \mathrm{e}^{i \omega(k) t}\left(-i \partial_{x}\right)^{j} q(\alpha, t) d t, \quad j=0, \ldots, n-1, \quad k \in \mathbb{C},
\end{aligned}
$$

где $\alpha=0, L$ и $D_{+}, D_{-}-$области, заданные определением 2.1 . 


\section{3. ГЛОБАЛЬНОЕ УСЛОВИЕ}

Общее описание шагов в рамках метода Фокаса, приводящих к представлению (2.2), можно найти в работах [1], [4]; в данной работе мы только подчеркнем, что доказательства сушественным образом используют некоторое глобальное алгебраическое соотношение, связывающее все граничные значения в определенных областях спектральной комплексной плоскости. Чтобы сделать это понятие точным, дадим определение набора допустимых функций; ими являются множества функций, которые могут возникать как граничные значения решения заданной корректно поставленной задачи с граничными условиями.

ОПРЕДЕЛЕНИЕ 3.1. Пусть $q_{0}(x) \in \mathbf{C}^{\infty}[0, L]$ и $\omega(k)$ определяется уравнением (1.3). Пусть $\left\{f_{0}(t), \ldots, f_{n-1}(t), g_{0}(t), \ldots, g_{n-1}(t)\right\}$ - набор $2 n$ достаточно гладких функций таких, что $\left(-i \partial_{x}\right)^{j} q_{0}(0)=f_{j}(0),\left(-i \partial_{x}\right)^{j} q_{0}(L)=g_{j}(0), j=0, \ldots, n-1$. Пусть

$$
\begin{aligned}
\widehat{F}(k) & =\sum_{j=1}^{n} \alpha_{j}\left(\hat{f}_{j-1}(k)+k \hat{f}_{j-2}(k)+\cdots+k^{j-1} \hat{f}_{0}(k)\right), \\
\widehat{G}(k) & =\sum_{j=1}^{n} \alpha_{j}\left(\hat{g}_{j-1}(k)+k \hat{g}_{j-2}(k)+\cdots+k^{j-1} \hat{g}_{0}(k)\right),
\end{aligned}
$$

где

$$
\begin{array}{ll}
\hat{f}_{j}(k)=\int_{0}^{T} \mathrm{e}^{i \omega(k) t} f_{j}(t) d t, & j=0, \ldots, n-1, \quad k \in \mathbb{C}, \\
\hat{g}_{j}(k)=\int_{0}^{T} \mathrm{e}^{i \omega(k) t} g_{j}(t) d t, & j=0, \ldots, n-1, \quad k \in \mathbb{C} .
\end{array}
$$

Набор гладких функций $\left\{f_{0}(t), \ldots, f_{n-1}(t), g_{0}(t), \ldots, g_{n-1}(t)\right\}$ называется допустимымм по отношению $\kappa q_{0}(x)$, если и только если функции $\widehat{F}(k)$ и $\widehat{G}(k)$ удовлетворяют соотношению

$$
\widehat{F}(k)-\mathrm{e}^{-i k L} \widehat{G}(k)=-\hat{q}_{0}(k)+\mathrm{e}^{i \omega(k) T} \hat{a}(k), \quad k \in \mathbb{C},
$$

где

$$
\hat{a}(k)=\int_{0}^{L} \mathrm{e}^{-i k x} a(x) d x
$$

а $a(x)$ - некоторая функция, принадлежащая пространству $\mathbf{C}^{\infty}[0, L]$.

В частном случае, когда функции $\left\{f_{0}(t), \ldots, f_{n-1}(t), g_{0}(t), \ldots, g_{n-1}(t)\right\}$ являются граничными значениями решения $q(x, t)$ уравнения $(1.1)$, а именно когда $f_{j}(t)=$ $\left(-\partial_{x}\right)^{j} q(0, t)$ и $g_{j}(t)=\left(-\partial_{x}\right)^{j} q(L, t)$, даваемая методом Фокаса конструкция автоматически приводит к тому, что они удовлетворяют уравнению $(3.5)$ при $a(x)=q(x, T)$; в этом случае об уравнении (3.5) говорят как о глобальном соотношении. 


\section{4. ВЫВОД О СУШЕСТВОВАНИИ РЕШЕНИЯ}

Ниже мы представим доказанный в работе [1] результат для частного случая, когда граничная задача для уравнения (1.1) получается путем предписывания первых $N$ производных функции $q(x, t)$ при $x=0$ и первых $n-N$ производных при $x=L$, т.е. для случая, когда заданные граничные условия имеют вид

$$
\begin{aligned}
& \left(-i \partial_{x}\right)^{j_{1}} q(0, t)=u_{j_{1}}(t), \quad 0<t<T, \quad 0 \leqslant j_{1} \leqslant N-1, \\
& \left(-i \partial_{x}\right)^{j_{2}} q(L, t)=v_{j_{2}}(t), \quad 0<t<T, \quad 0 \leqslant j_{2} \leqslant n-N-1,
\end{aligned}
$$

где $N$ определяется уравнением (1.5). Для формулировки этих результатов нам потребуются несколько предварительных определений.

ОПРЕДЕЛЕниЕ 4.1. Многочлены $\omega_{n-j}(k), j=1, \ldots, n$, определяются как

$$
\omega_{n-j}(k)=\alpha_{j}+k \alpha_{j+1}+\cdots+\alpha_{n} k^{n-j} .
$$

Пусть $D_{+}$и $D_{-}$определены выражением (2.1). Можно полагать, не теряя обшности, что $D_{+}$имеет $N$ односвязных компонент $\left\{D_{+, m}\right\}, m=1, \ldots, N$, а $D_{-}$имеет $n-N$ односвязных компонент $\left\{D_{-, l}\right\}, l=1, \ldots n-N$; более того, можно рассматривать отображение $\lambda_{l, m}: \bar{D}_{+, m} \rightarrow \bar{D}_{-, l}$ как биголоморфное отображение, определенное соотношением

$$
\omega\left(\lambda_{l, m}(k)\right)=\omega(k)
$$

для любого $k \in \bar{D}_{+, m}$ (см. [5]).

ОПРЕДЕЛЕНИЕ 4.2. А является $(n \times n)$-матрищей

$$
\left(\begin{array}{cccccc}
E_{1} \omega_{n-N-1}\left(\lambda_{1, m}\right) & \ldots & E_{1} \omega_{0}\left(\lambda_{1, m}\right) & -\omega_{N-1}\left(\lambda_{1, m}\right) & \ldots & -\omega_{0}\left(\lambda_{1, m}\right) \\
E_{2} \omega_{n-N-1}\left(\lambda_{2, m}\right) & \ldots & E_{2} \omega_{0}\left(\lambda_{2, m}\right) & -\omega_{N-1}\left(\lambda_{2, m}\right) & \ldots & -\omega_{0}\left(\lambda_{2, m}\right) \\
\cdot & \ldots & \cdot & \cdot & \ldots & \cdot \\
\cdot & \ldots & \cdot & \cdot & \ldots & \\
E_{N} \omega_{n-N-1}\left(\lambda_{N, m}\right) & \ldots & E_{N} \omega_{0}\left(\lambda_{N, m}\right) & -\omega_{N-1}\left(\lambda_{N, m}\right) & \ldots & -\omega_{0}\left(\lambda_{N, m}\right) \\
\omega_{n-N-1}\left(\lambda_{N+1, m}\right) & \ldots & \omega_{0}\left(\lambda_{N+1, m}\right) & -E_{N+1}^{-1} \omega_{N-1}\left(\lambda_{N+1, m}\right) & \ldots & -E_{N+1}^{-1} \omega_{0}\left(\lambda_{N+1, m}\right) \\
\cdot & \ldots & \cdot & \cdot & \ldots & \cdot \\
\cdot & \ldots & \cdot & \cdot & \ldots & \cdot \\
\omega_{n-N-1}\left(\lambda_{n, m}\right) & \ldots & \omega_{0}\left(\lambda_{n, m}\right) & -E_{n}^{-1} \omega_{N-1}\left(\lambda_{n, m}\right) & \ldots & -E_{n}^{-1} \omega_{0}\left(\lambda_{n, m}\right)
\end{array}\right)
$$

где $E_{l}=\mathrm{e}^{i \lambda_{l, m} L}$, а $\lambda_{l, m}=\lambda_{l, m}(k)$ - отображение, определяемое выражением (4.3); $b=$ $\left(b_{1}, \ldots, b_{N}, b_{N+1}, \ldots, b_{n}\right)^{\tau}-n$-вектор, компоненты которого определяются как

$$
b_{l}=\left\{\begin{array}{c}
\sum_{j=1}^{n-N} \omega_{n-j}\left(\lambda_{l, m}\right) \hat{g}_{j-1}(k)-\mathrm{e}^{i \lambda_{l, m} L}\left[\sum_{j=1}^{N} \omega_{n-j}\left(\lambda_{l, m}\right) \hat{f}_{j-1}(k)+\hat{q}_{0}\left(\lambda_{l, m}\right)\right], \\
1 \leqslant l \leqslant N, \\
\mathrm{e}^{-i \lambda_{l, m} L} \sum_{j=1}^{n-N} \omega_{n-j}\left(\lambda_{l, m}\right) \hat{g}_{j-1}(k)-\sum_{j=1}^{N} \omega_{n-j}\left(\lambda_{l, m}\right) \hat{f}_{j-1}(k)+\hat{q}_{0}\left(\lambda_{l, m}\right), \\
N+1 \leqslant l \leqslant n ;
\end{array}\right.
$$

$c\left(\lambda_{1, m}, \ldots, \lambda_{n, m}\right)-n$-вектор, компоненты которого

$$
c_{l}= \begin{cases}\mathrm{e}^{i \lambda_{l, m} L+i \omega(k) T} \hat{a}\left(\lambda_{l, m}\right), & 1 \leqslant l \leqslant N, \\ \mathrm{e}^{i \omega(k) T} \hat{a}\left(\lambda_{l, m}\right), & N+1 \leqslant l \leqslant n,\end{cases}
$$


где $\hat{a}(k)$ такое же, как в уравнении (3.6).

Используя данные вьше определения, можно сформулировать результат следующим образом.

ПРЕДЛОЖЕНИЕ 4.1. Пусть

$$
\begin{aligned}
f_{j}(t)=u_{j_{1}}(t), & 0 \leqslant j_{1} \leqslant N-1, \\
g_{l}(t)=v_{j_{2}}(t), & 0 \leqslant j_{2} \leqslant n-N-1,
\end{aligned}
$$

где $u_{j-1}(t), v_{j-2}(t)$ такие же, как в уравнении (4.1).

Тогда существуют гладкие функиии $\left\{f_{l}(t)\right\}, l=N, \ldots, n-1, u\left\{g_{m}(t)\right\}, m=$ $n-N, \ldots, n-1$, такие, что множсество $\left\{f_{j}(t), g_{j}(t)\right\}_{j=0}^{n-1}$ является набором допустимых функиий по отношению $к q_{0}(x)$. Существуют спектральнье функиии

$$
\begin{array}{ll}
\hat{f}_{j}(k)=\int_{0}^{T} \mathrm{e}^{i \omega(k) t} f_{j}(t) d t, & j=0, \ldots, n-1, \quad k \in \mathbb{C}, \\
\hat{g}_{j}(k)=\int_{0}^{T} \mathrm{e}^{i \omega(k) t} g_{j}(t) d t, & j=0, \ldots, n-1, \quad k \in \mathbb{C},
\end{array}
$$

связанные с этими допустимыми функииями.

Решение $q(x, t)$ допускает представление

$$
\begin{aligned}
q(x, t)=\frac{1}{2 \pi} & \left\{\int_{-\infty}^{\infty} \mathrm{e}^{i k x-i \omega(k) t} \hat{q}_{0}(k) d k+\right. \\
& \left.+\int_{\partial D_{+}} \mathrm{e}^{i k x-i \omega(k) t} \widehat{F}(k) d k+\int_{\partial D_{-}} \mathrm{e}^{i k(x-L)-i \omega(k) t} \widehat{G}(k) d k\right\},
\end{aligned}
$$

где $\omega(k)$ дается уравнением (1.3), а $D_{+}$и $D_{-}$определяются выражсением (2.1).

Это представление можно также записать в виде

$$
\begin{aligned}
q(x, t)=\frac{1}{2 \pi} & \left\{\int_{-\infty}^{\infty} \mathrm{e}^{i k x-i \omega(k) t} \hat{q}_{0}(k) d k+\right. \\
& +\int_{\partial D_{+}} \mathrm{e}^{i k x-i \omega(k) t} \sum_{j=1}^{N} \omega_{n-j}(k) \hat{f}_{j-1}(k) d k+ \\
& \left.+\int_{\partial D_{-}} \mathrm{e}^{i k(x-L)-i \omega(k) t} \sum_{j=1}^{n-N} \omega_{n-j}(k) \hat{g}_{j-1}(k) d k\right\}+ \\
& +\sum_{m=1}^{N} \sum_{j=1}^{n-N}\left[\int_{\partial D_{+, m}} \mathrm{e}^{i k x-i \omega(k) t} \frac{\operatorname{det} B_{j}\left(\lambda_{1, m}(k), \ldots, \lambda_{n, m}(k)\right)}{2 \pi \Delta\left(\lambda_{1, m}(k), \ldots, \lambda_{n, m}(k)\right)} d k-\right. \\
& \left.-i \sum_{h} \mathrm{e}^{i k_{h} x-i \omega\left(k_{h}\right) t} \frac{\operatorname{det} B_{j}\left(\lambda_{1, m}\left(k_{h}\right), \ldots, \lambda_{n, m}\left(k_{h}\right)\right)}{\Delta^{\prime}\left(\lambda_{1, m}\left(k_{h}\right), \ldots, \lambda_{n, m}\left(k_{h}\right)\right)}\right]+ \\
& +\sum_{l=N+1}^{n} \sum_{j=n-N+1}^{n}\left[\int_{\partial D_{-, l}} \mathrm{e}^{i k(x-L)-i \omega(k) t} \frac{\operatorname{det} B_{j}\left(\lambda_{1, l}(k), \ldots, \lambda_{n, l}(k)\right)}{2 \pi \Delta\left(\lambda_{1, l}(k), \ldots, \lambda_{n, l}(k)\right)} d k-\right.
\end{aligned}
$$




$$
\left.-i \sum_{h} \mathrm{e}^{i \tilde{k}_{h}(x-L)-i \omega\left(\tilde{k}_{h}\right) t} \frac{\operatorname{det} B_{j}\left(\lambda_{1, l}\left(\tilde{k}_{h}\right), \ldots, \lambda_{n, l}\left(\tilde{k}_{h}\right)\right)}{\Delta^{\prime}\left(\lambda_{1, l}\left(\tilde{k}_{h}\right), \ldots, \lambda_{n, l}\left(\tilde{k}_{h}\right)\right)}\right],
$$

где $\hat{f}_{j}, \quad 0 \leqslant j \leqslant N, u \hat{g}_{l}, \quad 0 \leqslant l \leqslant n-N$, даются уравнениями (4.7) $u(4.8) ; D_{+, m}-$ $N$ связанных компонент $D_{+}$, a $D_{-, l}-n-N$ связанных компонент $D_{-} ; \quad \Delta-$ определитель $(n \times n)$-матрицы $A$, задаваемой уравнением (4.4); $\left\{k_{h}\right\}-$ нули $\Delta в$ области $D_{R, m} ; \tilde{k}_{h}=\lambda_{l, m}\left(k_{h}\right) ; B_{j}\left(\lambda_{1, m}, \ldots, \lambda_{n, m}\right)$-матрица, полученная заменой $j$-го столбца матриць $A\left(\lambda_{1, m}, \ldots, \lambda_{n, m}\right)$ на вектор $b\left(\lambda_{1, m}, \ldots, \lambda_{n, m}\right)$, определенный в (4.5); $\Delta^{\prime}$ обозначает производную $\Delta$ по $k$.

Аналогичный результат получается для другого набора предписанных граничных условий, если то же число условий, что и в предложении 4.1, задается в двух граничных точках $x=0$ и $x=L$.

Основа доказательства состоит в том, чтобы показать, что заданные граничные условия достаточны для определения спектральных функций $\widehat{F}(k)$ и $\widehat{G}(k)$, в терминах которых выражается представление. Это можно получить, анализируя свойства ограниченности глобального соотношения на спектральной комплексной плоскости и используя биголоморфные отображения, определяемые уравнением (4.3) между односвязными областями $D_{+, m}$ и $D_{-, l}$. А именно, заметим, что уравнение (3.5) можно записать в эквивалентной форме

$$
\mathrm{e}^{i k L} \widehat{F}(k)-\widehat{G}(k)=-\mathrm{e}^{i k L} \hat{q}_{0}(k)+\mathrm{e}^{i k L+i \omega(k) T} \hat{a}(k) .
$$

Хотя оба соотношения корректно определены при всех $k \in \mathbb{C}$, при $k \rightarrow \infty$ уравнение (3.5) ограничено, только если $k \in D_{-}$, тогда как (4.11) ограничено, только если $k \in D_{+}$. Используя определение (3.1), (3.2) функций $\widehat{F}$ и $\widehat{G}$, а также глобальные соотношения (4.11) для $k \in D_{R,+}$ и $(3.5)$ для $k \in D_{R,-}$, можно доказать следующую лемму.

Лемма 4.1. Пусть заданьи $q_{0}(x) \in \mathbf{C}^{\infty}[0, L]$ и набор гладких функций $\left\{f_{j}(t), g_{j}(t)\right\}$, $0 \leqslant j \leqslant n-1$, являющихся допустимыми по отношению $\kappa q_{0}(x)$.

Для $k \in D_{+, m}, 1 \leqslant m \leqslant N$, функиии $\left\{\hat{f}_{j}(k), \hat{g}_{j}(k)\right\}, 0 \leqslant j \leqslant n-1$, определяемые соотношениями (3.3), (3.4), удовлетворяют системе уравнений

$$
\begin{aligned}
& \mathrm{e}^{i \lambda_{l, m} L} \sum_{j=1}^{n} w_{n-j}\left(\lambda_{l, m}\right) \hat{f}_{j-1}(k)-\sum_{j=1}^{n} w_{n-j}\left(\lambda_{l, m}\right) \hat{g}_{j-1}(k)= \\
& =-\mathrm{e}^{i \lambda_{l, m} L} \hat{q}_{0}\left(\lambda_{l, m}\right)+\mathrm{e}^{i \lambda_{l, m} L+i w(k) T} \hat{a}\left(\lambda_{l, m}\right), \quad 1 \leqslant l \leqslant N, \\
& \sum_{j=1}^{n} w_{n-j}\left(\lambda_{l, m}\right) \hat{f}_{j-1}(k)-\mathrm{e}^{-i \lambda_{l, m} L} \sum_{j=1}^{n} w_{n-j}\left(\lambda_{l, m}\right) \hat{g}_{j-1}(k)= \\
& =-\hat{q}_{0}\left(\lambda_{l, m}\right)+\mathrm{e}^{i w(k) T} \hat{a}\left(\lambda_{l, m}\right), \quad(N+1) \leqslant l \leqslant n,
\end{aligned}
$$

где многочлены $\omega_{n-j}(k)$ определяются вирахсением (4.2).

Перенося в правую часть линейной системы (4.12) известные функции $\hat{f}_{j}(k), j=$ $0, \ldots, N$ и $\hat{g}_{j}(k), j=0, \ldots, n-N$, получаем систему для оставшихся функций, входяших в определение спектральных функций $\widehat{F}(k), \widehat{G}(k)$. Требуется показать, что эта 
система имеет единственное решение, которое является множеством функций со свойствами аналитичности и ограниченности, связанными с тем, что эти функции возникают из множества гладких функций от $x$ и $t$ по формулам $(3.1),(3.2)$. А именно, $\widehat{F}(k)$ и $\widehat{G}(k)$ должны быть аналитическими функциями $k$, ограниченными при $k \rightarrow \infty$ в области $D$.

В работе [1] показано, что это имеет место для данного частного множества граничных условий, а в работе [2] - в обшем случае для любого набора из $N$ граничных условий, заданных при $x=0$, и $n-N$ граничных условий при $x=L$.

\section{5. НАБРОСОК ДОКАЗАТЕЛЬСТВА ТЕОРЕМЫ 1.1 И ПРЕДЛОЖЕНИЯ 1.1}

Из рассмотрения системы $n$ уравнений (4.12) ясно, что система, полученная путем переноса в правую часть каждого уравнения членов, содержащих соответствующие заданным условиям функции, в обшем случае допускает единственное решение, только если она содержит ровно $n$ неизвестных. Такая система в обшем случае имеет вид

$$
\begin{gathered}
\mathrm{e}^{i \lambda_{l, m} L} \sum_{j_{1} \in J_{1}^{\prime}} w_{n-j_{1}}\left(\lambda_{l, m}\right) \hat{f}_{j_{1}-1}(k)-\sum_{j_{2} \in J_{2}^{\prime}} w_{n-j_{2}}\left(\lambda_{l, m}\right) \hat{g}_{j_{2}-1}(k)= \\
=-\mathrm{e}^{i \lambda_{l, m} L} \sum_{j_{1} \in J_{1}} w_{n-j_{1}}\left(\lambda_{l, m}\right) \hat{f}_{j_{1}-1}(k)+\sum_{j_{2} \in J_{2}} w_{n-j_{2}}\left(\lambda_{l, m}\right) \hat{g}_{j_{2}-1}(k)- \\
\quad-\mathrm{e}^{i \lambda_{l, m} L} \hat{q}_{0}\left(\lambda_{l, m}\right)+\mathrm{e}^{i \lambda_{l, m} L+i w(k) T} \hat{a}\left(\lambda_{l, m}\right), \quad 1 \leqslant l \leqslant N, \\
\sum_{j_{1} \in J_{1}^{\prime}} w_{n-j_{1}}\left(\lambda_{l, m}\right) \hat{f}_{j_{1}-1}(k)-\mathrm{e}^{-i \lambda_{l, m} L} \sum_{j_{2} \in J_{2}^{\prime}} w_{n-j_{2}}\left(\lambda_{l, m}\right) \hat{g}_{j_{2}-1}(k)= \\
=-\sum_{j_{1} \in J_{1}} w_{n-j_{1}}\left(\lambda_{l, m}\right) \hat{f}_{j_{1}-1}(k)+\mathrm{e}^{-i \lambda_{l, m} L} \sum_{j_{2} \in J_{2}} w_{n-j_{2}}\left(\lambda_{l, m}\right) \hat{g}_{j_{2}-1}(k)- \\
\quad-\hat{q}_{0}\left(\lambda_{l, m}\right)+\mathrm{e}^{i w(k) T} \hat{a}\left(\lambda_{l, m}\right), \quad(N+1) \leqslant l \leqslant n,
\end{gathered}
$$

где $J_{i}^{\prime}=\{0, \ldots, n-1\} \backslash J_{i}, i=1,2$, и индексы $\left(j_{1}, j_{2}\right)$ принадлежат некоторому множеству индексов $J_{1} \times J_{2}, J_{i} \subset\{0, \ldots, n-1\}$, такому, что мошность $J_{1} \times J_{2}$ равна $n$. Возможность построения представления решения конкретной граничной задачи в виде (4.9) сводится, таким образом, к нахождению условий, обеспечивающих тот факт, что для заданного конкретного набора $n$ голоморфных ограниченных функций линейная система (5.1) имеет в качестве своего единственного решения набор из $n$ функций со следуюшими дополнительными свойствами: они являются голоморфными функциями от $k$ и ограничены при $k \rightarrow \infty$ в $D$.

Доказательство этого результата включает два основных шага.

Шаг 1. Докажем, что единственным случаем, приводяшим к системе, решение которой есть набор $n$ ограниченных в $D$ функций, является случай, когда матрица $A$, определяющая $(n \times n)$-систему, получается путем выбора $n-N$ столбцов среди первых $n$ столбцов $(n \times 2 n)$-системы $(4.12)$, определяюшей матрицу, и $N$ столбцов среди последних $n$ столбцов системы (4.12).

При любом другом выборе матрицы $A$ единственное решение этой системы не обладает требуемыми свойствами $k$-асимптотической ограниченности. 
ШШаг 2. Докажем, что для любой $(n \times n)$-системы, полученной описанным выше путем, решение является в обшем случае набором мероморфных функций, но сушествует подходяший выбор функции $a(x)$, при котором это решение действительно является набором голоморфных функций, ограниченных при $k \rightarrow \infty$ в $D$.

Доказательство шага 1 основано на том, какой вид принимает определитель системы по отношению к конкретному множеству неизвестных; в рассматриваемых случаях обшее экспоненциальное поведение ограничено в рассматриваемой области, в то время как при любом другом выборе можно показать, что результирующая экспонента не ограничена при $k \rightarrow \infty$ в $D$.

Шаг 2 доказывается с помощью такого специального выбора функции $a(x)$, чтобы вычесть вклад от полюсов определителя системы и таким образом получить набор голоморфных функций в качестве ее решения.

Сформулированные выше результаты позволяют доказать теорему 1.1 и предложение 1.1.

ДОКАЗАТЕЛЬСТВО ТЕОРЕМЫ 1.1 немедленно следует в предположении о справедливости результатов, сформулированных в шагах 1 и 2.

Действительно, пусть $\left|J_{1}\right|=N$ и $\left|J_{2}\right|=n-N$. Рассмотрим систему, получаемую выбором ровно $n-N$ столбцов среди первых $n$ в (4.12), соответствующих индексам из $J_{1}$, и $N$ столбцов среди последних $n$ в (4.12), соответствующих индексам из $J_{2}$. В силу шага 1 решение этой системы дается набором $n$ функций, ограниченных в $D$; обозначим их как $\left\{\hat{h}_{p}(k)\right\}_{p=1}^{n}$. Функции $\left\{\hat{h}_{p}(k)\right\}_{p=1}^{n}$ являются в общем случае мероморфными функциями $k$; однако в силу шага 2 можно выбрать функцию $a(x)$ так, что получаюшееся решение является набором голоморфных функций. Рассмотрим набор $\left\{\hat{f}_{0}, \ldots, \hat{f}_{n-1}\right.$, $\left.\hat{g}_{0}, \ldots, \hat{g}_{n-1}\right\}$, где

$$
\begin{array}{ll}
\hat{f}_{j_{1}}(k)=\int_{0}^{T} \mathrm{e}^{i \omega(k) t} u_{j_{1}}(t) d t, & j_{1} \in J_{1}, \\
\hat{g}_{j_{2}}(k)=\int_{0}^{T} \mathrm{e}^{i \omega(k) t} v_{j_{2}}(t) d t, & j_{2} \in J_{2},
\end{array}
$$

и $n$ остающихся пробелов в последовательности функций последовательно заполняются функциями $\hat{h}_{p}(k), p=1, \ldots, n$. По построению соответствующий набор $\left\{f_{0}, \ldots, f_{n-1}\right.$, $\left.g_{0}, \ldots, g_{n-1}\right\}$ является допустимым по отношению к $q_{0}(x)$. Отсюда в силу предложения 4.1 граничная задача имеет единственное решение с требуемым свойством гладкости.

ДоКАЗАТЕЛЬСТВО ПРЕДЛОЖЕНИЯ 1.1. ПредПоложим, что решение $q(x, t)$ данной задачи сушествует и имеет указанные свойства гладкости, так что данная граничная задача корректно поставлена. Граничные значения решения, т.е. множество функций $\left(-i \partial_{x}\right)^{j_{1}} q(0, t),\left(-i \partial_{x}\right)^{j_{2}} q(L, t), 0 \leqslant j_{i} \leqslant n-1$, являются набором допустимых функций по отношению к начальной функции $q_{0}(x)$; в частности, они удовлетворяют глобальным условиям (3.5) и (4.11) при $a(x)=q(x, T)$, а потому и системе (4.12), построенной с использованием этих условий. Перенося в правую часть системы члены, соответствующие данным граничным условиям, получаем $(n \times n)$-систему вида $(5.1)$, которой удовлетворяют функции $\hat{f}_{j_{1}}(k)=\widehat{Q}_{j_{1}}(0, k)$ и $\hat{g}_{j_{2}}(k)=\widehat{Q}_{j_{2}}(L, k)$, определенные в терминах 
граничных значений $q(x, t)$ с помощью уравнения (2.5). Более того, эти функции аналитичны по $k$ и ограничены при $k \rightarrow \infty$ в $D$. Такое возможно, только если матрица, определяюшая $(n \times n)$-систему $(5.1)$, получена выбором $n-N$ столбцов среди первых $n$ в (4.12) и $N$ столбцов среди последних $n$ в (4.12), т.е. только если $\left|J_{1}\right|=N$ и $\left|J_{2}\right|=n-N$.

\section{6. ЗАКЛЮЧЕНИЕ}

Представленные результаты получены применением метода преобразования Фокаса к граничным задачам для заданных на конечном интервале линейных эволюционных уравнений в одном пространственном измерении. Этот метод позволяет доказать, что задачи, полученные предписыванием определенного числа граничных условий на одном и на другом конше интервала, являются корректно поставленными. Кроме того, он дает представление решения, которое обладает явной зависимостью от $x$ и $t$, определяемой соответствуюшим дисперсионным соотношением; это представление содержит в общем случае интегральную часть и сумму по точкам из дискретного спектра соответствующего $x$-дифференциального оператора.

Заметим, что их можно использовать и для получения информации о нелинейных эволюционных дифференциальных уравнениях в частных производных в одном пространственном измерении, в то время как представленные здесь строгие результаты относятся только к линейнымм задачам.

Если нелинейное дифференциальное уравнение в частных производных является интегрируемым, то приведенный анализ линейной части может быть делинеаризован посредством анализа пары Лакса; в этом случае можно видеть, что тот же класс граничных задач корректно поставлен глобально по $t$. Пример нелинейного уравнения Шредингера на интервале $[0,1]$ проанализирован в работе [6]. Если нелинейное дифференциальное уравнение в частных производных не является интегрируемым, мы интерпретируем его нелинейную часть как вынуждающий член: таким образом, по крайней мере на малых временах, анализ линейной части любого заданного эволюционного уравнения дает результаты о корректности граничных задач на конечном интервале. Подобная же аргументация дается в работе [7] для систем дифференциальных уравнений в частных производных.

\section{Список литературы}

[1] A.S. Fokas, B. Pelloni. Math. Proc. Camb. Philos. Soc. 2001. V. 131. P. 521.

[2] B. Pelloni. Well posed boundary value problems for linear evolution equations on a finite interval. Math. Proc. Cam. Phil. Soc. (in press).

[3] A.S. Fokas. J. Math. Phys. 2000. V. 41. P. 4188.

[4] A.S. Fokas. Proc. Roy. Soc. London. A. 2001. V. 457. P. 371.

[5] A.S. Fokas, L. Y. Sung. Initial boundary value problems for linear evolution equations on the half line (to appear).

[6] A.S. Fokas, A.R. Its. The nonlinear Schrödinger equation on a finite interval (to appear).

[7] D. Antonopoulos, V.A. Dougalis, A.S. Fokas, B. Pelloni. Boundary value problems for Boussinesq type system. J. Math. Anal. Appl. 2002 (submitted). 